\title{
Probióticos en pediatría
}

\author{
Guillermo Álvarez Calatayud \\ Sección de Gastroenterología y Nutrición Pediátrica. Hospital General \\ Universitario Gregorio Marañón. Madrid. \\ Presidente de la Sociedad Española de Microbiota, Probióticos y Pre- \\ bióticos (SEMiPyP)

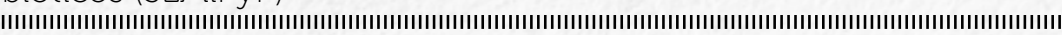

\section{Resumen}

La colonización microbiana del tracto digestivo durante la infancia es un proceso esencial para nuestra existencia. La implicación del microbioma en las causas y desarrollo de diversas enfermedades y su posible aplicación en el tratamiento de las mismas, con su modulación con el uso de probióticos y prebióticos, ha cobrado un inusitado interés científico en los últimos años, afirmación avalada por numerosos proyectos de investigación. En pediatría, cada vez se da más importancia al uso de probióticos y prebióticos a cualquier edad para tratar diversas enfermedades, principalmente problemas gastrointestinales, aunque también se utilizan con éxito en alergias y en la prevención de infecciones. Al mismo tiempo, la investigación está abriendo la puerta a futuras aplicaciones como la obesidad y los trastornos del comportamiento, como el autismo.

Palabras clave: Probióticos, prebióticos, microbiota intestinal, pediatría, disbiosis, ensayos clínicos

\section{Probiotics in pediatrics}

\section{Abstract}

Microbial colonization of the digestive tract during infancy is an essential process for our existence. The implication of the microbiome in the causes and development of various diseases and its possible application in their treatment, with its modulation with the use of probiotics and prebiotics, has gained unusual scientific interest in recent years, a claim supported by numerous research projects. In pediatrics, increasing importance is given to the use of probiotics and prebiotics at any age to treat various diseases, mainly gastrointestinal problems, although they are also used successfully in allergies and in the prevention of infections. At the same time, research is opening the door to future applications such as obesity and behavioral disorders such as autism.

Key words: Probiotics, prebiotics, gut microbiota, pediatrics, dysbiosis, clinical trials.

\section{Introducción}

Cada individuo posee una comunidad microbiana peculiar que depende de su genotipo y de la exposición temprana a los microorganismos de su entorno, pero también de la dieta, los cambios de estilo de vida o la terapia frente a las infecciones. Esto implica que la colonización desde el nacimiento será diferente dependiendo de factores como el tipo de parto, del modelo de lactancia, el entorno rural o urbano en que crecemos, el nacer en un país en vías de desarrollo o desarrollado, el uso de antibióticos, especialmente los utilizados para combatir infecciones durante el parto y en la primera infancia, etc. Un inadecuado desarrollo de nuestra microbiota digestiva durante los primeros meses de vida por el aumento del número de cesáreas, el abandono prematuro de la lactancia materna o, ya en la edad adulta, por el abuso de antibióticos, una dieta inadecuada o el proceso del envejecimiento, nos puede llevar a un estado de disbiosis con una alteración de la microbiota tanto cualitativa (especies distintas a las habituales) como cuantitativa (menor concentración de sus bacterias beneficiosas). La consecuencia será la disminución de sus efectos saludables $y$, con frecuencia, la aparición de enfermedades como, por ejemplo, las diarreas $^{1}$.

En 2001, una comisión de expertos internacionales convocados de forma conjunta por la FAO y la OMS definió a los probióticos como "microorganismos vivos que cuando se administran en cantidades adecuadas confieren un beneficio a la salud del hospedador". Desde entonces, esta definición ha sido la más ampliamente aceptada en todo el mundo y ha sido recientemente confirmada por la ISAPP (International Scientific Association of Probiotics and Prebiotics $^{2}$. Los organismos probióticos remedarían los efectos positivos que ejerce la microbiota autóctona, por lo que, en principio, cualquier componente de ella podría ser candidato a convertirse en probiótico. 
Sin embargo, la mayoría pertenecen a dos grupos microbianos: los lactobacilos y las bifidobacterias, aunque la levadura Saccharomyces cerevisiae y algunas especies de $E$. coli y Bacillus también son empleadas. Los prebióticos son ingredientes alimentarios que producen una estimulación selectiva del crecimiento y/o actividad(es) de uno o de un limitado número de géneros/especies de microorganismos en la microbiota intestinal confiriendo beneficios para la salud del hospedador ${ }^{3}$. Como prebióticos más conocidos nos encontramos los fructooligosacáridos (FOS), los galactooligosacáridos (GOS), la inulina, la lactulosa y, por supuesto, los oligosacáridos de la leche materna. Se denomina simbiótico al producto que combina al menos un probiótico y un prebiótico. Si el carbohidrato prebiótico es utilizado por la cepa probiótica, se promoverá selectivamente su proliferación en el intestino del hospedador. Es interesante señalar que la combinación de probiótico y prebiótico en un mismo producto puede tener un efecto sinérgico y conferir beneficios mayores que los que ejercen cada uno de los componentes del simbiótico por separado ${ }^{4}$. Recientemente se ha introducido el término postbiótico al referirse a sustancias producidas por los probióticos que ejercen efectos metabóli- $\cos y / 0$ inmunomoduladores en el huésped sin que por el momento haya tenido repercusiones importantes tanto desde el punto de vista investigador como clínico ${ }^{5}$. Los psicobióticos serían organismos vivos que, cuando se consume en cantidades adecuadas, producen un beneficio en la salud de pacientes con trastornos psiquiátricos $^{6}$. En la tabla I se incluyen las definiciones de la familia de los bióticos con la finalidad de clarificar la nomenclatura y evitar confusiones?

Cada vez se da mayor importancia a la modulación de la microbiota intestinal mediante el empleo de probióticos, prebióticos y simbióticos para tratar diversas enfermedades, principalmente problemas gastrointestinales, como diferentes tipos de diarreas (de la comunidad, asociadas a los antibióticos, del viajero, intolerancia a la lactosa, etc.), trastornos funcionales (cólico del lactante o síndrome del intestino irritable) o procesos inflamatorios (colitis ulcerosa). Además, se usan con éxito en diversas patologías de la mujer (vulvovaginitis y mastitis) y se ha valorado su efecto en las alergias como la dermatitis atópica y en la prevención de infecciones (desde el recién nacido prematuro hasta el anciano $)^{7}$. Paralelamente a los numerosos pro-

Tabla I. Definiciones. Tomada de Alvarez-Calatayud G, Leis R, Díaz JJ, 2021, con autorización 7

PROBIÓTICOS (Lilly-Stillwell, 1965)

Microorganismos vivos que confieren un beneficio a la salud del huésped cuando se los administra en cantidades adecuadas.

PREBIÓTICOS (Gibson-Roberfroid, 1995)

Ingredientes fermentados selectivamente que dan lugar a cambios específicos en la composición y/o actividad de la microbiota gastrointestinal, confiriendo así beneficios a la salud del huésped.

SIMBIÓTICOS (Gibson-Roberfroid, 1995)

Productos que contienen tanto probióticos como prebióticos.

POSTBIÓTICOS (Rescigno, 2012)

Compuestos microbianos generados por el metabolismo de los probióticos y liberados al medio extracelular que, cuando son administrados en cantidades adecuadas, producen un efecto beneficioso para el consumidor.

PARAPROBIÓTICOS (Taverniti, 2011)

Células microbianas inactivadas o fracciones de células que cuando son administrados en cantidades adecuadas, pueden conferir un beneficio para el consumidor.

PSICOBIOTICOS (Dinan, 2013)

Organismos vivos que, cuando se consumen en cantidades adecuadas, producen un beneficio en la salud de pacientes con trastornos mentales. 
yectos de investigación que han aparecido en los últimos años dedicados a ampliar el conocimiento de la microbiota autóctona, las investigaciones en animales de experimentación y en humanos abren la puerta a futuras aplicaciones con la suplementación de estos organismos y nutrientes. Hay estudios que apoyarían su utilización en problemas nutricionales (obesidad, malnutrición), enfermedades neurológicas y alteraciones del comportamiento (autismo, depresión, ansiedad), en la enfermedad periodontal y la eventual prevención en varios tipos de cáncer. Por último, se está avanzando en las repercusiones que la alteración de la microbiota puede tener en la aparición de enfermedades cardiovasculares, arterioesclerosis, diabetes, hipercolesterolemia, síndrome metabólico, etc. ${ }^{8}$.

La Pediatría es una de las disciplinas donde los metanálisis y revisiones sistemáticas de ensayos clínicos contrastados han demostrado su eficacia en diversas patologías, sobre todo digestivas. Por este motivo, su empleo se va asentando paulatinamente en la práctica clínica habitual de los pediatras. Además, existen varias líneas de investigación abiertas en la suplementación con probióticos en la infancia. A continuación, vamos a desarrollar las principales patologías pediátricas donde las principales guías de práctica clínica recomiendan su empleo basándose en la evidencia científica existente hasta el momento ${ }^{9}$.

Aplicaciones clinicas de los probióticos

\section{Prevención de la enterocolitis necrotizante}

La mayoría de metanálisis que han estudiado el uso de probióticos en recién nacidos tienen como objetivo primario la valoración de su empleo en la prevención de la enterocolitis necrotizante (NEC). Según éstos, la suplementación enteral con algunas cepas reduce de forma significativa frente al placebo la incidencia de NEC grave (estadios II o mayores) e incluso en algunos de ellos apuntan a un descenso de la mortalidad. Sin embargo, no se ha observado una disminución significativa en la sepsis nosocomial o en los días de nutrición parenteral. El efecto beneficioso de los probióticos en la madurez gastrointestinal y en su función podría mejorar la tolerancia enteral así como reducir el riesgo de enterocolitis necrotizante en los pretérmino. Algunos ensayos han analizado también este punto, observando que los neonatos con probióticos alcanzaban nutriciones enterales completas significativamente antes que en los grupos control. Se necesitan más ensayos para evaluar este potencial beneficio de los probióticos, en vista del papel importante de la intolerancia enteral y la consecuente privación nutricional en la restricción postnatal al crecimiento de los recién nacidos con extremo bajo peso ${ }^{10,11}$. A la vista de los múltiples ensayos clínicos que muestran efectos beneficiosos de los probióticos y la escasez de datos que los relacionan con efectos adversos graves, parece que la evidencia actualizada apoya fuertemente un cambio en la práctica clínica e indica que existe seguridad y eficacia en el uso de los probióticos, incluso en extremos prematuros (los de mayor riesgo de $\mathrm{NEC}$, muerte e intolerancia alimentaria).

\section{Probióticos y prebióticos en las fórmulas lác- teas infantiles}

Las fórmulas lácteas surgen como una alternativa en la nutrición del lactante cuando la lactancia materna no es posible. El objetivo final de las fórmulas lácteas infantiles es acercarse al "patrón oro", la leche materna, no sólo copiando sus componentes, sino intentando conseguir una funcionalidad similar, medible por sus efectos en el lactante. De hecho, el perfil microbiano de los recién nacidos a término, por parto vaginal y alimentados exclusivamente con leche matera constituye el estándar de microbiota beneficiosa y debe servir de referencia para el desarrollo de las fórmulas infantiles. La composición de las fórmulas se ha ido modificando a medida que se han ido conociendo las necesidades nutricionales del lactante. Desde hace muchos años se sabe que la leche materna evoluciona y cambia continuamente, adaptándose a las necesidades de cada niño a lo largo de la lactancia, y que mantiene unas diferencias básicas respecto a la leche de vaca, que es desde la que partimos para la elaboración de las fórmulas lácteas. Los tres componentes (probióticos, prebióticos y simbióticos) pueden ser añadidos a las fórmulas lácteas infantiles ${ }^{12}$.

Estas fórmulas lácteas son seguras y han demostrado ser bien toleradas por los lactantes, no producen efectos adversos y no alteran el crecimiento. Además, presentan una serie de efectos clínicos 
beneficiosos tales como menor incidencia de infecciones gastrointestinales y de vías respiratorias altas, menor utilización de antibióticos, menos incidencia de procesos alérgicos, principalmente dermatitis atópica, en los lactantes con riesgo y menor incidencia de estreñimiento. Aun así, el Comité de Nutrición de la Sociedad Europea de Gastroenterología, Hepatología y Nutrición Pediátrica (ESPGHAN) no recomienda el empleo de fórmulas suplementadas con probióticos y/o prebióticos de forma rutinaria, aunque es posible que la adición de determinadas cepas pueda ser recomendable. La escasa calidad de los estudios metodológicos (tipo de probiótico, duración de la intervención) hacen que el Comité crea que aún hay demasiada incertidumbre. Aun así, debido a que son necesarios más estudios con mayor rigor metodológico, no recomienda el uso sistemático de una fórmula suplementada con estos componentes en lactantes ${ }^{13}$.

\section{Diarrea aguda infecciosa}

La Organización Mundial de Gastroenterología (WGO), en su última actualización en 2017 ha ampliado significativamente, a la vista de los ensayos clínicos realizados, el número de preparados con probióticos para la diarrea infantil, aunque sigue remarcando que las cepas con mayor nivel de evidencia científica son Lactobacillus rhamnosus GG y Saccharomyces boular$d_{i i{ }^{14}}$. La última revisión de la GPC del Grupo de Trabajo de Probióticos y Prebióticos de la ESPGHAN ha rebajado el grado de recomendación, pero sigue afirmando que las cepas con evidencia científica suficiente siguen siendo S. boulardii, L. rhamnosus GG y, en menor medida, Lactobacillus reuteri DSM $17938{ }^{15}$. En general, la eficacia de los probióticos en el tratamiento de la diarrea aguda en los niños se produce al acortar en un día la duración del episodio, disminuir el número de deposiciones al segundo día de tratamiento y el porcentaje de diarreas que se prolongan más de 4 días. Parece que los efectos beneficiosos son más notables cuanto más precozmente se administran los probióticos en el curso de la enfermedad, no evidenciándose efectos adversos con su administración. El efecto beneficioso es más significativo en las diarreas producidas por rotavirus.

\section{Diarrea asociada a antibióticos}

El consumo de antibióticos es muy frecuen- te en los países desarrollados siendo en los niños tres veces mayor al de la población adulta. La antibioterapia puede alterar la resistencia a la colonización de la microbiota intestinal, dando lugar a una diversidad de síntomas, entre los que destaca la diarrea. Denominamos diarrea asociada a antibióticos (DAA) a aquélla que aparece desde el inicio del tratamiento antibiótico hasta 3-8 semanas después, siendo inexplicable por otra causa. Su incidencia es de un $11-40 \%$ en los niños, según las diferentes estadísticas y prácticamente todos los antibióticos pueden desencadenarla, aunque es más frecuente con aquéllos de amplio espectro ${ }^{16}$. Los ensayos realizados han podido demostrar que los probióticos en combinación con antibióticos reducen el riesgo de diarrea asociada a los mismos, aunque no hay, por el momento, estudios concluyentes para recomendar de manera rutinaria el empleo conjunto de probióticos y antibióticos. Sin embargo, algunas cepas, principalmente $S$. boulardii y $L$. rhamnosus GG, han demostrado su eficacia disminuyendo la incidencia de DAA. Su administración debe realizarse al principio del tratamiento y no cuando se ha desencadenado el cuadro y prolongarse durante todo el tratamiento antibiótico ${ }^{17}$.

\section{Trastornos funcionales digestivos en pedia- tría}

Los probióticos podrían actuar, por un lado, sobre la alteración de microbiota y por otro mejorando la motilidad intestinal, modificando la percepción de dolor intestinal por inhibición de la contractilidad intestinal y disminuyendo la inflamación intestinal, así como inhibiendo el crecimiento de bacterias coliformes productoras de gas en niños con cólicos. Se han publicado numerosos ensayos clínicos sobre diferentes probióticos en el cólico del lactante, sobre todo de L. reuteri DSM17398, con buenos resultados por lo que esta cepa está recomendada en la última revisión de la guía de la WGO tanto en el tratamiento como en su prevención ${ }^{14,18}$.

En relación al estreñimiento, el empleo de prebióticos aumenta la capacidad de retención de agua de las heces y estimula el crecimiento de las bifidobacterias, aumentando la media de deposiciones y disminuyendo su consistencia. La inulina y los fructooligosacáridos (FOG) poseen efectos laxantes dosis-dependiente que se atribu- 
yen al aumento de la biomasa microbiana como resultado de su fermentación en el colon. En un estudio en lactantes en los que se evaluó el empleo de oligosacárido de fructosa controlado con placebo, se demostró que el uso de prebióticos era efectivo con un aumento significativo del número de deposiciones y una disminución de su consistencia ${ }^{19}$.

La eficacia de los probióticos en el dolor abdominal funcional y en el síndrome del intestino irritable (SII) se basaría en sus propiedades sobre la homeostasis intestinal, con mantenimiento de la barrera intestinal y modulación de respuesta inmunológica innata y adquirida intestinal y sistémica. Los metanálisis más recientes corroboran que los probióticos disminuyen los síntomas del SII con ciertas cepas únicas o combinadas. Algunas cepas probióticas han demostrado que son más eficaces que el placebo en el tratamiento de los pacientes pediátricos con trastornos funcionales asociados a dolor abdominal especialmente en el SII. Prueba de ello se refleja en las recomendaciones de la última y reciente revisión de la GPC sobre Probióticos y Prebióticos de la Organización Mundial de Gastroenterología (WGO) ${ }^{14}$.

\section{Probióticos en el tratamiento de la enferme- dad inflamatoria intestinal}

En la enfermedad inflamatoria intestinal (EII), los resultados de ensayos clínicos con probióticos son contradictorios. Ninguno ha demostrado su eficacia en la enfermedad de Crohn, mientras que, en colitis ulcerosa, parece que el uso de VSL\#3 contribuyó a inducir y mantener la remisión. Las Guías Europeas de la ECCO (European Crohn's and Colitis Organization) reservan un posible papel terapéutico a los probióticos en determinadas indicaciones en la Ell. Parece evidente que éste es un campo prometedor y a desarrollar. Son necesarios más estudios para valorar qué especies y cepas y qué dosis deben ser utilizadas ${ }^{20}$.

\section{Enfermedad celíaca}

Los niños que padecen enfermedad celíaca presentan mayor número de bacterias Gram negativas y menor número de Gram positivas en su microbiota. Además, en el postratamiento hay menos lactobacilos, enterococos y bifidobacterias, más diversidad de eubacterias y más bacteroides, estafilococos, Salmonella, Shigella y Klebsiella que en niños sanos. Parece que sería necesaria una dieta sin gluten durante al menos dos años para restaurar la microbiota y el metaboloma de estos niños. Sin embargo, se han observado alteraciones en la microbiota no solo en pacientes celíacos no tratados, sino también en algunos que siguen una dieta libre de gluten. Asimismo, algunos niños celiacos continúan con síntomas, a pesar de recibir dieta libre de gluten y normalizar su mucosa intestinal. Estos hallazgos sugieren que la disbiosis podría asociarse a la persistencia de síntomas gastrointestinales en pacientes celíacos tratados, abriendo así nuevas perspectivas al tratamiento de este tipo de pacientes ${ }^{21}$.

\section{Malabsorción de la lactosa}

La microbiota intestinal juega también un importante papel en la tolerancia e intolerancia de los que maldigieren la lactosa, al condicionar la compensación colónica, uno de los factores determinantes de la sintomatología. Los probióticos en derivados lácteos fermentados podrían mejorar la tolerancia a la lactosa modulando la microbiota intestinal, favoreciendo la compensación colónica, así como su degradación por las enzimas bacterianas. El panel de expertos de la EFSA ha encontrado evidencia en 15 de los 18 estudios científicos presentados para afirmar que las bacterias del yogur vivas (Lactobacillus bulgaricus y Streptococcus thermophilus) mejoran la digestión de la lactosa en individuos con intolerancia ${ }^{22}$. De hecho, el uso de microorganismos del yogur para mejorar la intolerancia a la lactosa es, por el momento, la única declaración de propiedades saludables aceptada por el marco regulador de la CE. Una reciente revisión sistemática respalda los efectos beneficiosos de la suplementación con probióticos en los resultados de la prueba de $\mathrm{H} 2$ en el aliento y en los síntomas de la intolerancia a la lactosa ${ }^{23}$.

\section{Infección por Helicobacter pylori}

Como tratamiento coadyuvante de los regímenes de erradicación de $H$. pylori, los probióticos podrían disminuir los efectos secundarios del tratamiento y mejorar las tasas de erradicación. Además, por diferentes mecanismos inmunológicos y no inmunológicos, como la producción de sustancias antimicrobianas (ácidos gra- 
sos de cadena corta y bacteriocinas), los probióticos competirían por los sitios de unión al receptor de adhesión de $H$. pylori en las células epiteliales, fortaleciendo la barrera de la mucosa gástrica (estimulando la producción de mucina) y modulando la respuesta inmune frente a los patógenos.

Diferentes metanálisis y estudios sobre probióticos y $\mathrm{H}$. pylori han demostrado que la administración de las cepas Lactobacillus casei DN-114 001 y S. boulardii CNCM 1-745, según la revisión de 2017 de la guía de la $\mathrm{WGO}^{14}$, pueden mejorar, como terapia coadyuvante, las tasas de erradicación y disminuir los efectos adversos de la antibioterapia. Pueden ser especialmente útiles en los pacientes con infección por $\mathrm{H}$. pylori refractaria a tratamientos previos o antecedentes de mala tolerancia gastrointestinal de los antibióticos. Dada la evidencia disponible, en la cuarta edición del consenso de Maastricht en el tratamiento de la infección por $H$. pylori se declaró que "ciertos probióticos muestran resultados prometedores como tratamiento coadyuvante en la reducción de los efectos secundarios" 24.

\section{Alergia a proteínas de la leche de vaca}

El tratamiento de la alergia a las proteínas de la leche de vaca (APLV) se basa en la eliminación del alergeno sospechoso (proteínas de la leche de vaca) con el empleo de fórmulas lácteas sustitutivas, generalmente fórmulas de alto grado de hidrólisis. Es, precisamente en este tipo de leches especiales, donde se suplementa con probióticos y prebióticos para evitar los síntomas de la APLV y adquirir la tolerancia inmunológica. Aunque todavía está por determinar el papel que jugaría su empleo en esta patología y son necesarios más estudios para evaluar su eficacia, empiezan a aparecer numerosos ensayos clínicos controlados con diferentes cepas con resultados esperanzadores por lo que su uso va teniendo aceptación en las GPC ${ }^{25}$.

\section{Dermatitis atópica}

Aunque poco estudiada hasta este momento, la microbiota de la piel, seguramente proteja a este órgano y sus anejos de muchas de sus enfermedades $y$, probablemente contribuya al mantenimiento de la salud más allá del ámbito dérmico. Es posible que alguno de los microbios de nuestra piel pueda estar implicado en la etiología de muchos procesos dermatológicos como la dermatitis atópica. Precisamente es por lo que se empiezan a emplear probióticos para el tratamiento de diversas afecciones dermatológicas tanto en preparados orales como por vía tópica. Seguramente los estudios con mayor evidencia científica apuntan a su administración en la prevención de la dermatitis atópica temprana en niños con alto riesgo de padecerla, siendo el Lactobacillus rhamnosus GG la cepa más estudiada. Aun así, la mayoría de las GPC opinan que son necesarios más ensayos clínicos bien diseñados antes de poder recomendar su empleo de manera rutinaria ${ }^{26}$.

\section{Prevención de infecciones}

Una reciente revisión sistemática del uso de probióticos en la prevención de las infecciones respiratorias de vías altas pone en evidencia que en la mayoría de los estudios se observa al menos un efecto beneficioso. Incluso una reducción mínima del 5-10\% en la incidencia de estas infecciones tendría un importante impacto clínico y económico en la sociedad. Además, la administración a largo plazo de los probióticos parece ser segura en niños y ninguno de los estudios ha reportado efectos adversos serios relacionados con la cepa probiótica. Sin embargo, son necesarios más estudios para recomendar los probióticos como prevención de infecciones extraintestinales ${ }^{27}$.

\section{Obesidad}

Estudios recientes relacionan el parto por cesárea con disbiosis en los niños, por retraso y reducción en la exposición a la colonización microbiana y, por tanto, mayor riesgo de desarrollo de obesidad en edades posteriores. El uso y abuso de antibióticos en diversas etapas de la vida (embarazo, nacimiento, lactancia y primera infancia) provoca una disbiosis de la microbiota intestinal con un incremento de proteobacterias y otras poblaciones anaeróbicas (enterobacterias, clostidios y bacteroides) y disminución de la microbiota beneficiosa (bifidobacterias y lactobacilos) lo que unido a otros factores (genéticos, dieta, estilo de vida, etc.) puede llegar a ser un riesgo potencial del desarrollo posterior de sobrepeso y obesidad. Las poblaciones de Bifidobacterium y Staphylococcus aureus en el niño son posibles factores predictivos 
inversos y directos del desarrollo de obesidad en la edad adulta ${ }^{28}$.

\section{Trastornos del espectro autista (TEA)}

El uso de dietas especiales, probióticos y prebióticos o del trasplante fecal abren una puerta a la esperanza del beneficio que supone la modificación de la microbiota intestinal en los trastornos neurológicos y conductuales, como el TEA. Los probióticos y prebióticos podrían mejorar no sólo los síntomas gastrointestinales de estos pacientes, sino también las alteraciones del comportamiento. De hecho, hay varios meta-análisis que han evaluado la eficacia de los probióticos y prebióticos en estos pacientes con mejoras en sus síntomas digestivos, así como en los atributos conductuales de la escala de evaluación de la gravedad del autismo ATEC, como el lenguaje, la sociabilidad, el aprendizaje o el comportamiento. ${ }^{29}$ Se han desarrollado ensayos en estos pacientes utilizando un trasplante fecal con buena seguridad y excelentes resultados. ${ }^{30}$ Aunque, en general, se han sugerido resultados prometedores, se necesitan más ensayos clínicos bien diseñados, aleatorizados y controlados con placebo para validar la eficacia de los probióticos, los prebióticos o el trasplante fecal en el tratamiento del TEA para identificar las cepas, las dosis y la duración del tratamiento adecuadas. Por ello, es necesario ser cautos, ya que uno de los principales objetivos en estos niños y adolescentes es mejorar su calidad de vida y no debemos caer en el error de crear falsas esperanzas a los pacientes y a las familias. En el trastorno por déficit de atención e hiperactividad (TDAH) también se han relacionado los cambios en la composición de la microbiota intestinal con el desarrollo de esta patología, aunque son necesarios más estudios para confirmar esta hipótesis ${ }^{31}$.

\section{Conclusiones}

La microbiota intestinal está condicionada por factores genéticos y ambientales y la disbiosis puede ser causa de patología digestiva y extradigestiva a corto, medio y largo plazo, habiendo muchos estudios que evidencian el efecto positivo del uso de probióticos y prebióticos sobre la salud en niños, especialmente en relación con la inmunidad y la inflamación. El efecto del probiótico debe valorarse en función de la cepa utilizada, la dosis, el tiempo de admi- nistración y la duración del mismo. En el tratamiento de la diarrea aguda infecciosa y en la prevención de la diarrea asociada a antibióticos se ha demostrado que el empleo de los probióticos tiene el mayor nivel de evidencia. La inclusión de probióticos y prebióticos en las Guías de Práctica Clínica de patologías en el niño y el adolescente requieren de más estudios aleatorizados y de seguimiento, con un tamaño muestral adecuado, que demuestren su eficacia y seguridad a largo plazo.

\section{Bibliografía}

1. Matamoros S, Gras-Leguen C, Le Vacon F, Potel G, de La Cochetiere MF. Development of intestinal microbiota in infants and its impact on health. Trends in Microbiology 2013; 21 (4): 167-175.

2. Hill C, Guarner F, Reid G, Gibson GR, Merenstein DJ, Pot B, Morelli L, Canani RB, Flint HJ, Salminen S, Calder PC, Sanders ME. Expert consensus document. The International Scientific Association for Probiotics and Prebiotics consensus statement on the scope and appropriate use of the term probiotic. Nat Rev Gastroenterol Hepatol. 2014 Aug; 11(8):50614.

3. Gibson GR, Hutkins R, Sanders ME, Prescott SL, Reimer RA, Salminen SJ, et al. The International Scientific Association for Probiotics and Prebiotics (ISAPP) consensus statement on the definition and scope of prebiotics. Nat Rev Gastroenterol Hepatol. 2017; 14: 491502.

4. Swanson KS, Gibson GR, Hutkins R, Reimer RA, Reid G, Verbeke K. et al. The International Scientific Association for Probiotics and Prebiotics (ISAPP) consensus statement on the definition and scope of synbiotics. Nat Rev Gastroenterol Hepatol 2020. https://doi. org/10.1038/s41575-020-0344-2.

5. Salminen S, Collado MC, Endo A, Hill C, Lebeer $S$, Quigley EMMet al. The International Scientific Association for Probiotics and Prebiotics (ISAPP) consensus statement on the definition and scope of postbiotics. Nat Rev Gastroenterol Hepatol 2021. https://www.nature. com/articles/s41575-021-00440-6

6. Dinan TG, Satanton C, Cryan JF. Psychobiotics: a novel class of psychotropic. Biol Psychiatry. 2013; 74: 720-726.

7. Alvarez-Calatayud G, Leis R, Díaz JJ. Modulación de la microbiota intestinal. Uso de probióticos y prebióticos en pediatría. En: SEGHNP (Ed.). Tratamiento en Gastroenterología, Hepatología y Nutrición Pediátrica. 5a ed. Madrid: Ergon; 2021. p. 783-797.

8. Rodriguez JM. Probiotics: from the lab to the consumer. Nutr Hosp 2015; 31 S1: s33-s47.

9. Pérez Moreno J, Maturana D, Álvarez-Calatayud G, Bodas A. Guías de práctica clínica para el empleo de probióticos y prebióticos. En: Álvarez-Calatayud G, Marcos A, Margollés A (Eds.). Probióticos, prebióticos y salud: Evidencia científica. Madrid: Ergon; 2016. p. 375- 
393.

10. AlFaleh K, Anabrees J. Probiotics for prevention of necrotizing enterocolitis in preterm infants. Cochrane Database Syst Rev. 2014 Apr 10;(4):CD005496.

11. van den Akker CHP, van Goudoever JB, Shamir R, Domellöf M, Embleton ND, Hojsak I, et al. Probiotics and Preterm Infants: A Position Paper by the European Society for Paediatric Gastroenterology Hepatology and Nutrition Committee on Nutrition and the European Society for Paediatric Gastroenterology Hepatology and Nutrition Working Group for Probiotics and Prebiotics. Journal of pediatric gastroenterology and nutrition. 2020;70(5):66480.

12. Maldonado Lozano J. Nuevos ingredientes en las fórmulas para lactantes nacidos a término (I): Probióticos, prebióticos y simbióticos. Acta Pediatr Esp. 2014, 72: 56-62.

13. ESPGHAN Committee on Nutrition. Supplementation of infant formula with probiotics and/or prebiotics: a systematic review and comment by the ESPGHAN Committee on Nutrition JPGN 2011; 52: 238-250.

14. Guarner F, Sanders ME, Kaufmann P, de Paula JA, Fedorak R, Garisch J et al; World Gastroenterology Organization. World Gastroenterology Organisation Global Guidelines: probiotics and prebiotics. February 2017. (www.worldgastroenterology.org/probiotics-prebiotics. html).

15. Szajewska H, Guarino A, Hojsak I, Indrio F, Kolacek S, Orel R et al. On behalf of the Working Group on Probiotics and Prebiotics of the European Society for Paediatric Gastroenterology, Hepatology and Nutrition. Use of Probiotics for the Management of Acute Gastroenteritis in Children: An Update. J Pediatr Gastroenterol Nutr 2020; 71: 261-269.

16. Johnston BC, Goldenberg JZ, Vandvik PO, Sun $X$, Guyatt GH. Probiotics for the prevention of pediatric antibiotic-associated diarrhea. Cochrane Database Syst Rev. 2011; (11): CD004827.

17. Szajewska H, Canani RB, Guarino A, Hojsak I, Indrio $F$, Kolacek $S$ et al. on Behalf of the ESPGHAN Working Group for Probiotics/Prebiotics. Probiotics for the Prevention of Antibiotic-Associated Diarrhea in Children. J Pediatr Gastroenterol Nutr. 2016. 62(3):495-506.

18. Szajewska H, Gyrczuk E, Horvath A. Lactobacillus reuteri DSM 17938 for the management of infantile colic in breastfed infants: a randomized, double-blind, placebo-controlled trial. J Pediat 2013;162: 257-262.

19. Moore N, Chao C, Yang L, et al. Effects of fructo-ologosaccharide-supplemented cereal: a double blind placebo controlled multinational study. Br JNutr 2003; 90:581-587.

20. Miele E, Shamir R, Aloi M, Assa A, Braegger C, Bronsky J, et al. Nutrition in Pediatric Inflammatory Bowel Disease: A Position Paper on Behalf of the Porto Inflammatory Bowel Disease Group of the European Society of Pediatric Gastroenterology, Hepatology and Nutrition. Journal of pediatric gastroenterology and nutrition. 2018;66(4):687-708.

21. Olivares $M$, Walker AW, Capilla A, Benítez-Páez A, Palau F, Parkhill J et al. Gut microbiota tra- jectory in early life may predict development of celiac disease. Microbiome 2018; 6:36. https://doi.org10.1186/s40168-018-0415-6.

22. Oak SJ, Jha R. The effects of probiotics in lactose intolerance: A systematic review. Crit Rev Food Sci Nutr. 2019; 59:1675-1683.

23. Leis R, de Castro MJ, de Lamas C, Picáns R, Couce ML. Effects of Prebiotic and Probiotic Supplementation on Lactase Deficiency and Lactose Intolerance: A Systematic Review of Controlled Trials. Nutrients 2020, 12, 1487; doi:10.3390/nu12051487.

24. Malfertheiner $P$, Megraud F, O'Morain CA, Gisbert JP, Kuipers EJ, Axon AT et al. Management of Helicobacter pylori infection-the Maastricht V/Florence Consensus Report. Gut. 2016; 0: 1-25.

25. Muraro A, Halken S, Arshad SH, Beyer K, Dubois AE, Du Toit G, et al. EAACI Food Allergy and Anaphylaxis Guidelines Group. EAACl Food Allergy and Anaphylaxis Guidelines. Primary prevention of food allergy. Allergy. 2014; 69(5):590-601.

26. Sidbury R, Tom WL, Bergman JN, Cooper KD, Silverman RA, Berger TG, et al. Guidelines of care for the management of atopic dermatitis. Section 4. Prevention of disease flares and use of adjunctive therapies and approaches. J Am Acad Dermatol. 2014; 71(6):1218-1233.

27. Hao Q, Dong BR, Wu T. Probiotics for preventing acute upper respiratory tract infections. Cochrane Database Syst Rev. 2015:CD006895.

28. Rodriguez JM, Sobrino OJ, Marcos A, Collado MC, Pérez G, Martinez MC et al. ¿Existe una relación entre la microbiota intestinal, el consumo de probióticos y la modulación del peso corporal?.Nutr Hosp 2013;28 S1:s3-s12.

29. Xu M, Xu X, Li J, Li F. Association between gut microbiota and autism spectrum disorder: A systematic review and meta-analysis. Front Psychiatry 2019; 10: 473.

30. Kang DK, Adams JB, Gregory AC, Borody T, Chittick L, Fasano A, et al. Microbiota Transfer Therapy alters gut ecosystem and improves gastrointestinal and autism symptoms: an open-label study. Microbiome. 2017; 5: 10.

31. Cenit MC, Campillo I, Codoñer-Franch P, Dinan TG, Sanz Y. Gut Microbiota and Attention Deficit Hyperactivity Disorder: New Perspectives for a Challenging Condition. European Child\&Adolescent Psychiatry 2017; 26 (9), 1081-1092. 